\title{
First-Principles Surface Phase Diagram for Hydrogen on GaN Surfaces
}

\author{
Chris G. Van de Walle ${ }^{1,2,3}$ and J. Neugebauer ${ }^{2}$ \\ ${ }^{1}$ Xerox Palo Alto Research Center, 3333 Coyote Hill Road, Palo Alto, California 94304 \\ ${ }^{2}$ Fritz-Haber-Institut der Max-Planck-Gesellschaft, Faradayweg 4-6, D-14195 Berlin-Dahlem, Germany \\ ${ }^{3}$ Paul-Drude-Institut, Hausvogteiplatz, 5-7, D-10117 Berlin, Germany
}

(Received 27 August 2001; published 28 January 2002)

\begin{abstract}
We discuss the derivation and interpretation of a generalized surface phase diagram, based on firstprinciples density-functional calculations. Applying the approach to hydrogenated GaN surfaces, we find that the Gibbs free energies of relevant reconstructions strongly depend on temperature and pressure. Choosing chemical potentials as variables results in a phase diagram that provides immediate insight into the relative stability of different structures. A comparison with recent experiments illustrates the power of the approach for interpreting and predicting energetic and structural properties of surfaces under realistic growth conditions.
\end{abstract}

DOI: $10.1103 /$ PhysRevLett.88.066103

A detailed understanding of semiconductor surface reconstructions is essential for controlling growth and materials properties. In the case of $\mathrm{GaN}$, where materials quality is still limiting device progress, the investigations until now have focused on reconstructions of bare GaN surfaces [1]. The present study focuses on the role of hydrogen $(\mathrm{H})$, which is important because $\mathrm{H}$ is abundantly present in the most commonly used growth techniques for nitride semiconductors, including metal-organic chemical vapor deposition (MOCVD), hydride vapor-phase epitaxy (HVPE), and molecular-beam epitaxy (MBE) when $\mathrm{NH}_{3}$ is used as the nitrogen source.

We have therefore performed detailed investigations of $\mathrm{H}$ interactions with GaN surfaces based on state-of-the-art pseudopotential-density-functional calculations. We focus on the technologically most relevant $\mathrm{GaN}(0001)$ surface, which is the polarity observed during MOCVD of GaN on sapphire as well as MBE on Si-face SiC. Since we are particularly interested in consequences for growth, it is essential to take finite temperature effects into account, requiring the calculation of free energies. This distinguishes our approach from previous work in which only zerotemperature energies were calculated, and then only for a small number of structures [2-4]. We will show that the energetic and structural features of the surface reconstructions dramatically depend on temperature $(T)$ and pressure $(p)$.

In thermochemistry, the Gibbs free energy $G$ is usually expressed as a function of $T$ and partial pressures. An alternative approach is to use chemical potentials $\mu$ as variables and to express $G$ as a function of $p, T$, and all independent $\mu$ 's. For the system at hand, the Gibbs' Phase Rule produces four degrees of freedom, resulting in a four-dimensional phase space that is difficult to analyze or visualize. Our detailed analysis will show that the explicit dependence on $p$ and $T$ is small and can be neglected. The surface energy can then be expressed solely as a function of the chemical potentials $\mu_{\mathrm{Ga}}$ and $\mu_{\mathrm{H}}$, while the $p$ and $T$ dependence is implicit. The advan-
PACS numbers: 68.35.-p, 68.43.-h, 81.10.-h

tage of this methodology is its physically intuitive character. Comparison with experiment requires only that the chemical potentials be evaluated as a function of $T$ and partial pressures. The power of this approach will become evident when we use it to interpret the recent in situ observations of surface reconstructions based on grazing incidence x-ray scattering by Munkholm et al. [5]. We will show that their measured transition between two reconstructions coincides with a specific boundary in our phase diagram, offering direct information about the nature of the observed structures.

Our first-principles approach is based on densityfunctional theory in the local-density approximation (LDA) [6], using ab initio pseudopotentials [7]. Test calculations using the generalized gradient approximation produced results in close agreement with LDA. The Ga $3 d$ states are explicitly included as valence states, requiring a 70 Ry plane-wave cutoff. We use a slab geometry, consisting of at least four double layers of GaN plus at least $7 \AA$ of vacuum. The lower surface of the slab is passivated with fractionally charged $\mathrm{H}$, and the positions of all atoms in the lower half of the slab are kept fixed. Convergence tests indicated that these choices allow us to determine energy differences with an accuracy of better than $0.05 \mathrm{eV}$.

We choose the ideal, bare $1 \times 1$ surface (relaxed but unreconstructed) as our reference, and define the Gibbs free energy of formation $\Delta G^{f}$ as follows:

$$
\begin{aligned}
\Delta G^{f}= & E_{\mathrm{tot}}[\mathrm{GaN}(0001)]-E_{\mathrm{tot}}[\mathrm{GaN}(0001), \text { ideal }] \\
& +\Delta F_{\mathrm{vib}}-n_{\mathrm{Ga}} \mu_{\mathrm{Ga}}-n_{\mathrm{N}} \mu_{\mathrm{N}}-n_{\mathrm{H}} \mu_{\mathrm{H}} .
\end{aligned}
$$

$E_{t}[\mathrm{GaN}(0001)]$ is the calculated total energy for the surface under study, and $E_{\text {tot }}[\mathrm{GaN}(0001)$, ideal $]$ is the total energy of our reference system. $n_{\mathrm{Ga}(\mathrm{N}, \mathrm{H})}$ is the number of $\mathrm{Ga}(\mathrm{N}, \mathrm{H})$ atoms added to the unit cell (positive or negative). $\Delta F_{\text {vib }}$ includes vibrational contributions to the free energy and is discussed below. $\mu_{\mathrm{Ga}}$ and $\mu_{\mathrm{N}}$ 
are the chemical potentials of $\mathrm{Ga}$ and $\mathrm{N}$, i.e., the free energies of the reservoirs with which $\mathrm{Ga}$ and $\mathrm{N}$ atoms are exchanged. Invoking equilibrium with $\mathrm{GaN}$ [8] leaves us with a single parameter to describe the stoichiometry, for which we choose $\mu_{\mathrm{Ga}} . \mu_{\mathrm{Ga}}$ varies over the thermodynamically allowed range: $\mu_{\mathrm{Ga}[\text { bulk }]}+\Delta H_{f}[\mathrm{GaN}]<\mu_{\mathrm{Ga}}<$ $\mu_{\mathrm{Ga}[\mathrm{bulk}]}$, the upper limit corresponding to Ga-rich conditions, the lower limit to N-rich $\left(\mu_{\mathrm{N}}=\mu_{\mathrm{N}\left[\mathrm{N}_{2}\right]}\right)$. $\Delta H_{f}[\mathrm{GaN}]$ is the enthalpy of formation (negative for a stable compound). Our calculated value for $\Delta H_{f}[\mathrm{GaN}]$ is $-1.24 \mathrm{eV}$ (experiment: $-1.17 \mathrm{eV}$, Ref. [9]). $\mu_{\mathrm{H}}$, finally, is the free energy of $\mathrm{H}_{2}$ and describes the abundance of $\mathrm{H}$ in the environment.

Figure 1(a) shows our calculated formation energies as a function of $\mu_{\mathrm{Ga}}$ at $T=0$. In total, we examined over 30 different structures; Fig. 1 includes only those reconstructions (schematically illustrated in Fig. 2) that were found to be energetically favorable in some part of the phase space spanned by the chemical potentials. In the absence of $\mathrm{H}$, we reproduce the results for bare $\mathrm{GaN}(0001)$ surfaces reported in Ref. [1]; specifically, under moderately Ga-rich conditions the most favorable reconstruction is the $\mathrm{Ga}$ adatom in a $T 4$ position (i.e., above a second-layer $\mathrm{N}$ atom), while under $\mathrm{N}$-rich conditions the $\mathrm{N}$ adatom in the $H 3$ position (i.e., the "hollow site" above the hexagonal channel) is most stable. Under extreme Ga-rich conditions, a Gabilayer reconstruction is energetically most favorable [10].

Although we made no a priori assumptions, we find a posteriori that the lowest-energy structures all obey electron counting [11]: any $\mathrm{N}$ dangling bonds (dbs) that remain on the surface after formation of $\mathrm{N}-\mathrm{H}$ or $\mathrm{Ga}-\mathrm{H}$ bonds are filled with two electrons, while Ga dangling bonds are empty. We actually examined many structures with $1 \times 1$ and $\sqrt{3} \times \sqrt{3}$ periodicity, in addition to the $2 \times 2$ structures reported in Fig. 1 . Only the $2 \times 2$ structures were energetically favorable, consistent with the easily derived result that the electron counting rule can be satisfied for tetrahedral coordination only if the unit cell contains a multiple of four surface atoms.

Figure 1(a) shows that at $T=0$ hydrogenated surfaces with a large number of N-H bonds have significantly lower energies than the bare surfaces. This is consistent with the notion that $\mathrm{N}-\mathrm{H}$ bond formation is favorable due to the large bond strength. In particular, the $\mathrm{NH}_{3}+3 \mathrm{NH}_{2}$ structure is favored over almost the entire range of $\mu_{\mathrm{Ga}}$; this structure is formed by adding one $\mathrm{NH}_{3}$ and three $\mathrm{NH}_{2}$ molecules in on-top positions (see Fig. 2). Alternatively, one can think of this structure as a $\mathrm{N}$-terminated $\mathrm{GaN}(0001)$ structure, with 9 of the $12 \mathrm{~N}$ dbs passivated by $\mathrm{H}$; this number of $\mathrm{H}$ atoms allows for the electron counting rule to be satisfied. Under extreme Ga-rich conditions, the $\mathrm{NH}_{3}+3 \mathrm{Ga}-\mathrm{H}$ structure is favorable; here, one $\mathrm{NH}_{3}$ unit sits on top of one of the Ga surface atoms, and $\mathrm{Ga}-\mathrm{H}$ bonds are formed at the other three $\mathrm{Ga}$ atoms in the cell. This structure is very close in energy to a stoichiometrically equivalent structure, namely, $V_{\mathrm{Ga}}+6 \mathrm{H}$, which con-
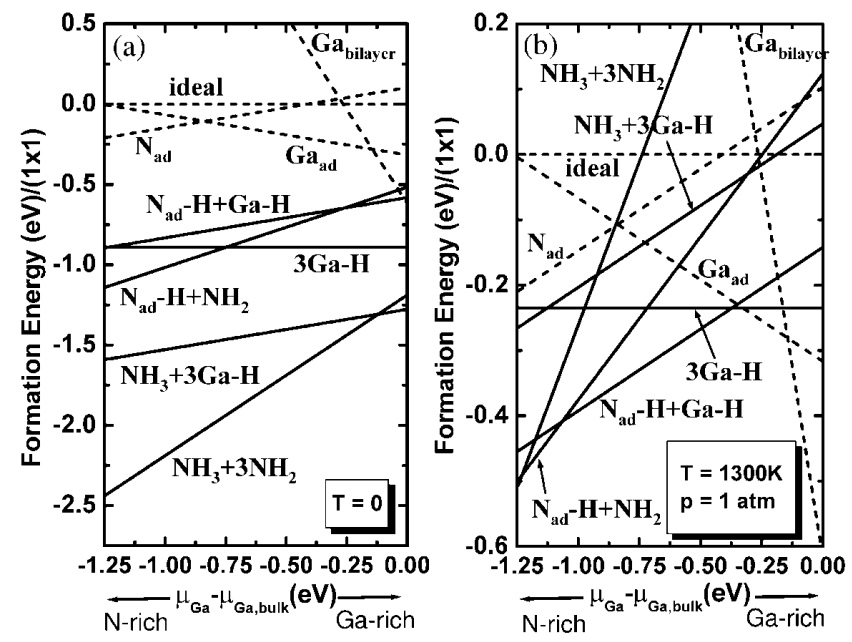

FIG. 1. Calculated formation energies for $\mathrm{GaN}(0001)$ surfaces, as defined in Eq. (1), as a function of $\mu_{\mathrm{Ga}}$. All structures (except $\mathrm{Ga}_{\text {bilayer }}$ ) have $2 \times 2$ periodicity, but energies are expressed in $\mathrm{eV}$ per $1 \times 1$ cell. Dashed lines indicate hydrogen-free surfaces. (a) $T=0$; (b) $T=1300 \mathrm{~K}, p\left(\mathrm{H}_{2}\right)=1 \mathrm{~atm}$. Note the very different energy scale in the two plots.

sists of a Ga vacancy in which all three $\mathrm{N}$ dbs are saturated with $\mathrm{H}$, plus three $\mathrm{Ga}-\mathrm{H}$ bonds.

If we limited our investigation to the $T=0$ results depicted in Fig. 1(a), we would conclude that the $\mathrm{NH}_{3}+$ $3 \mathrm{NH}_{2}$ structure, which includes the largest number of $\mathrm{N}-\mathrm{H}$ bonds, is dominant on the hydrogenated $\mathrm{GaN}(0001)$ surface. We will see, however, that this conclusion is not valid at higher temperatures if finite-temperature effects are incorporated in the formation energy defined in Eq. (1). The temperature dependence arises from vibrational contributions to the energy and entropy, and can, in principle, be evaluated entirely from first principles based on a calculation of the vibrational spectrum. Inspection of the vibrational modes calculated for a subset of structures reveals, however, that $\Delta F_{\text {vib }}$ in Eq. (1) can be calculated by assuming specific frequencies for the vibrational modes of the bonds that participate in these surface reconstructions [12]. Assuming that vibrational frequencies do not change by more than $\pm 100 \mathrm{~cm}^{-1}$ due to changes in the environment, we obtain a conservative error bar of less than $0.1 \mathrm{eV}$

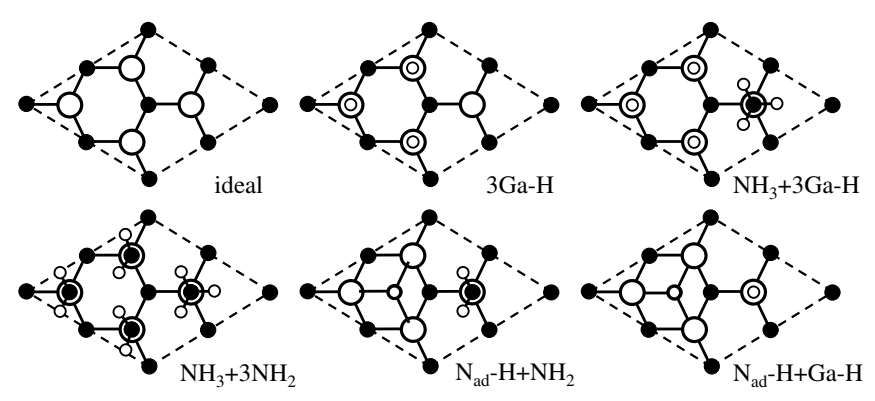

FIG. 2. Schematic top view of prevalent $2 \times 2$ reconstructions for $\mathrm{GaN}(0001)$ surfaces. Large open circles represent Ga atoms, solid circles $\mathrm{N}$, and small open circles $\mathrm{H}$. 
on the free energies calculated using this approximation. The temperature (and pressure) dependence of the reservoirs has also been included in Eq. (1); this is particularly important for gaseous species, e.g., for hydrogen:

$2 \mu_{\mathrm{H}}=E_{\mathrm{H}_{2}}+k T\left[\ln \left(\frac{p V_{Q}}{k T}\right)-\ln Z_{\mathrm{rot}}-\ln Z_{\mathrm{vib}}\right]$,

where $E_{\mathrm{H}_{2}}$ is the energy of an $\mathrm{H}_{2}$ molecule, $k$ is the Boltzmann constant, $T$ is the temperature, and $p$ is the pressure. $V_{Q}=\left(h^{2} / 2 \pi m k T\right)^{3 / 2}$ is the quantum volume, and $Z_{\mathrm{rot}}$ and $Z_{\mathrm{vib}}$ are the rotational and vibrational partition functions. The temperature dependence of $\mu_{\mathrm{H}}$ for two different pressures is shown in Fig. 3(b).

Figure 1(b) shows the formation energies calculated at $T=1300 \mathrm{~K}$ (a typical MOCVD growth temperature) and $p\left(\mathrm{H}_{2}\right)=1 \mathrm{~atm}$. Not only are the energetically favored reconstructions entirely different from those at $T=0$, but the energy difference between hydrogenated and bare surfaces has been dramatically reduced. While at a low temperature several $\mathrm{eV}$ could be gained by forming $\mathrm{N}-\mathrm{H}$ bonds, at a high temperature the energy difference between hydrogenated and bare surfaces has been reduced to less than $0.3 \mathrm{eV}$ - with the $\mathrm{H}$-free $\mathrm{Ga}_{\mathrm{ad}}$ and $\mathrm{Ga}_{\text {bilayer }}$ structures actually being favored under $\mathrm{Ga}$-rich conditions. The main reason for this dramatic difference is the large change in the value of $\mu_{\mathrm{H}}$, i.e., the free energy of the reservoir with which $\mathrm{H}$ atoms are exchanged: at $1300 \mathrm{~K} \mu_{\mathrm{H}}$ is about $1 \mathrm{eV}$ lower than at $T=0$ [Eq. (2) and Fig. 3(b)]. A lower value of $\mu_{\mathrm{H}}$ means that less energy can be gained by taking a $\mathrm{H}$ atom out of the reservoir and binding it to the $\mathrm{GaN}$

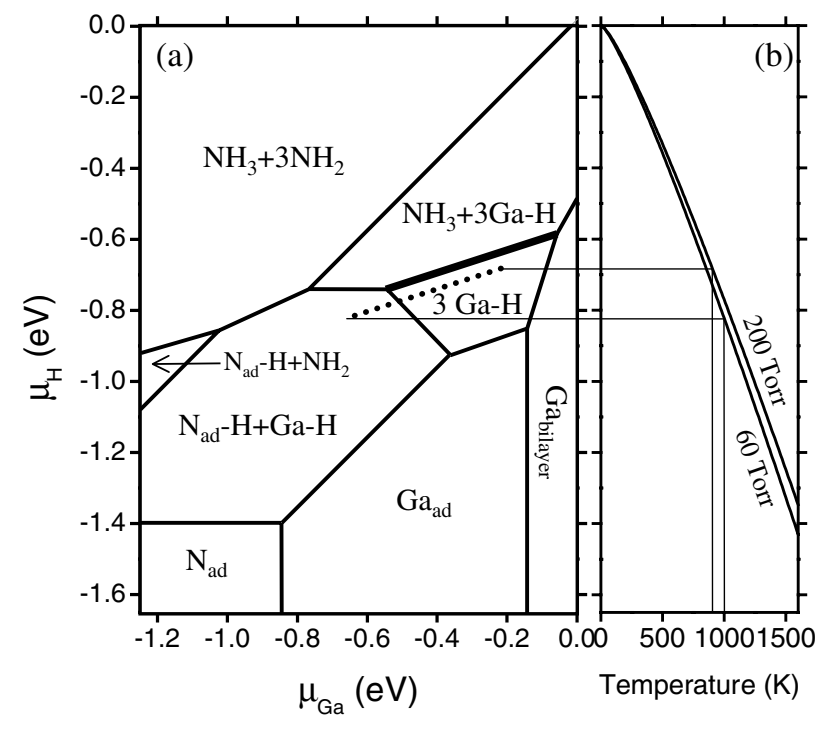

FIG. 3. (a) Phase diagram for the $\mathrm{GaN}(0001)$ surface in the presence of $\mathrm{H}$, as a function of $\mu_{\mathrm{Ga}}$ and $\mu_{\mathrm{H}} \cdot \mu_{\mathrm{H}}=0$ corresponds to $\mathrm{H}_{2}$ molecules at $T=0 ; \mu_{\mathrm{Ga}}=0$ corresponds to bulk Ga. Dots indicate experimental data from Ref. [5]; within the error bars, these data agree with the calculated $\mathrm{NH}_{3}+$ $3 \mathrm{Ga}-\mathrm{H} / 3 \mathrm{Ga}-\mathrm{H}$ phase boundary highlighted by the thicker line. Note that the $V_{\mathrm{Ga}}+6 \mathrm{H}$ structure is stoichiometrically and energetically equivalent to $\mathrm{NH}_{3}+3 \mathrm{Ga}-\mathrm{H}$. (b) Temperature dependence of $\mu_{\mathrm{H}}$ for two different pressures [Eq. (2)]. surface. The vibrational contributions to the free energy included in $\Delta F_{\text {vib }}$ are significantly smaller, on the order of $0.1 \mathrm{eV}$ at $T=1300 \mathrm{~K}$.

Changes in $T$ (and $p$ ) clearly have dramatic effects on the relative stability of different structures. As mentioned in the introduction, a traditional phase diagram would reflect four degrees of freedom, based on $\Delta G^{f}\left(p, T, p_{\mathrm{Ga}}\right.$, $\left.p_{\mathrm{H}}\right)$. The four-dimensional nature of the diagram presents a serious obstacle to straightfoward interpretation of the first-principles results. We now show that the number of degrees of freedom can be reduced to two if we choose the chemical potentials as variables, as shown in Fig. 3(a). The diagram shown here was calculated at $T=950 \mathrm{~K}$ (in order to facilitate the comparison with experiment discussed below), but the key point is that the important qualitative features of the diagram are independent of temperature and pressure. Small quantitative shifts in the positions of the boundaries do occur, but these amount to only about $0.1 \mathrm{eV}$ along either $\mu_{\mathrm{H}}$ or $\mu_{\mathrm{Ga}}$. Obviously, to obtain rigorous numbers one can refer back to Eq. (1) and take the detailed $T$ and $p$ dependence into account - but important qualitative insights can reliably be obtained from Fig. 3(a), even at temperatures significantly different from $T=950 \mathrm{~K}$.

The reasons why the number of degrees of freedom can be reduced from four to two are as follows. First, for the system at hand the total pressure plays a negligible role. As already implied in Eq. (1), the $p \Delta V$ term can be safely neglected in the free energy for the surface. In addition, the chemical potentials depend only on the respective partial pressures. Dropping the temperature as an explicit degree of freedom is based on the more approximate argument that the vibrational free energy contributions to $\Delta G$ are small, and exhibit significant cancellation when comparing $\Delta G$ for different reconstructions; the net effect of temperature on the phase boundaries is therefore quite small. We can thus write $\Delta G^{f}\left(p, T, p_{\mathrm{Ga}}, p_{\mathrm{H}}\right) \approx$ $\Delta G^{f}\left[\mu_{\mathrm{Ga}}\left(p_{\mathrm{Ga}}, T\right), \mu_{\mathrm{H}}\left(p_{\mathrm{H}}, T\right)\right]$. The remaining two variables, $\mu_{\mathrm{Ga}}$ and $\mu_{\mathrm{H}}$, are of course functions of $T$ and $p$, but otherwise the phase diagram exhibits no explicit $T$ or $p$ dependence. We suggest that this approach is quite general in nature. Hydrogenated $\mathrm{GaN}$ is actually a fairly extreme case, with both very high frequencies (N-H bonds, contributing to sizable vibrational energies) and very low frequencies (torsion modes, contributing to sizeable vibrational entropies). The success of the approach for this extreme case indicates it could be usefully applied to other systems as well.

We now discuss how Fig. 3 can be used to relate surface structures to experimental growth conditions. At the bottom of the diagram (low $\mu_{\mathrm{H}}$ ) we find the structures that dominate when little or no $\mathrm{H}$ is present. At the top of the diagram $\left(\mu_{\mathrm{H}}=0\right)$, we find the structures that were stable at $T=0$ [see Fig. 1(a)]. A horizontal line at $\mu_{\mathrm{H}}=-0.98 \mathrm{eV}$ would cut through the structures that were found to be stable at $1300 \mathrm{~K}$ and $p\left(\mathrm{H}_{2}\right)=1 \mathrm{~atm}$ [Fig. 1(b)]. The temperature dependence of $\mu_{H}$ is shown 
in Fig. 3(b), for two different pressures. Figure 3 makes it straightforward to investigate which structures would be stabilized by raising or lowering the pressure or the temperature.

Munkholm et al. [5], performed in situ grazing incidence $\mathrm{x}$-ray scattering on $\mathrm{GaN}(0001)$ surfaces in an MOCVD environment. Starting from $2-\mu$ m-thick GaN films grown on sapphire, they grew a 30-nm-thick layer at $1000^{\circ} \mathrm{C}$, on which they then performed their search for surface reconstructions. During this search, the sample was held at temperatures up to $1000{ }^{\circ} \mathrm{C}$, and $p\left(\mathrm{NH}_{3}\right)$ was varied between 2 and 140 Torr. The flow rate of the $\mathrm{NH}_{3}$ /carrier gas mixture was kept constant, with a total pressure of 200 Torr. Results obtained with $\mathrm{N}_{2}$ as the carrier gas were very similar to those obtained for $\mathrm{H}_{2}$.

At high temperatures, only a $1 \times 1$ reconstruction was observed. At lower temperatures and sufficiently low $\mathrm{NH}_{3}$ pressures, a different reconstruction was seen, which was identified as $(\sqrt{3} \times 2 \sqrt{3}) R 30^{\circ}$. The transition between the two reconstructions was mapped out as a function of $T$ and $p\left(\mathrm{NH}_{3}\right)$. From the temperature dependence of $p\left(\mathrm{NH}_{3}\right)$ at the transition, an apparent activation energy of $3.0 \pm 0.2 \mathrm{eV}$ was extracted. We now show that this experimentally established activation energy can be directly correlated with the energy difference between two surface reconstructions.

The experimental observation that the phase boundary is insensitive to whether $\mathrm{H}_{2}$ or $\mathrm{N}_{2}$ is used as the carrier gas indicates that the transformation between the two observed phases occurs purely through equilibration with $\mathrm{NH}_{3}$. The equilibrium expression $\mu_{\mathrm{N}}+3 \mu_{\mathrm{H}}=\mu_{\mathrm{NH}_{3}}$ then immediately fixes the slope of the phase boundary, already severely restricting the reconstructions that could be involved in the experimental transition. The position of the boundary is further determined by using the actual value $\mu_{\mathrm{NH}_{3}}=-2.28 \pm 0.2 \mathrm{eV}$ along the experimental phase boundary (referenced to the energy of the $\mathrm{NH}_{3}$ molecule), and has been included in Fig. 3(a). The end points correspond to the temperatures and pressures at the limits of the range explored in Ref. [5], assuming $\mathrm{H}_{2}$ as the carrier gas. Within the experimental [5] and computational uncertainty, we find good agreement with the calculated $\mathrm{NH}_{3}+3 \mathrm{Ga}-\mathrm{H} / 3 \mathrm{Ga}-\mathrm{H}$ phase boundary, including the fact that at temperatures above those reported in Ref. [5] this particular phase boundary should disappear because another reconstruction $\left(\mathrm{N}_{\mathrm{ad}}-\mathrm{H}+\mathrm{Ga}-\mathrm{H}\right)$ becomes more favorable.

The experimental observations are therefore consistent with a transition between two structures that differ only in the addition/subtraction of one $\mathrm{NH}_{3}$ unit in the surface unit cell. The $3 \mathrm{Ga}-\mathrm{H}$ structure (corresponding to a $3 / 4$ monolayer coverage of $\mathrm{H}$ ) is expected to exhibit $1 \times 1$ symmetry, since the experiment is not sensitive to $\mathrm{H}$ atoms [5]; in addition, disorder (due to different $\mathrm{H}$ positions in each $2 \times 2$ cell) would reduce the symmetry to $1 \times 1$. As for the other reconstruction, we suggest it is unlikely that it would indeed exhibit a $(\sqrt{3} \times 2 \sqrt{3}) R 30^{\circ}$ symmetry, as proposed in Ref. [5], since such a unit cell would not contain a multiple of four atoms and therefore would be energetically highly unfavorable, according to our first-principles results. The reconstruction peaks observed in Ref. [5] in fact exhibited $(2 \sqrt{3} \times 2 \sqrt{3}) R 30^{\circ}$ symmetry, and the reduction to $(\sqrt{3} \times 2 \sqrt{3}) R 30^{\circ}$ was based on a fit. A $(2 \sqrt{3} \times 2 \sqrt{3}) R 30^{\circ}$ reconstruction could be constructed out of $2 \times 2$ motifs, with interactions between the adspecies in neighboring $2 \times 2$ units stabilizing the structure. Explicit investigations of structures with 12 atoms in the surface unit cell are beyond the scope of the present investigations, but we suggest that the $\mathrm{NH}_{3}+3 \mathrm{Ga}-\mathrm{H}$ structure (and/or the $V_{\mathrm{Ga}}+6 \mathrm{H}$ structure, which is stoichiometrically and energetically equivalent) is a basic building block of the larger reconstruction.

In summary, we have outlined a computational approach for obtaining phase diagrams for systems consisting of an impurity-covered surface in thermodynamic contact with a gaseous environment, as characteristic of MOCVD or HVPE. The approach gives immediate access to the atomic structure and phase transitions of surfaces under realistic growth conditions, as illustrated with the important example of hydrogenated GaN surfaces. The methodology can be easily generalized to surface alloying or oxidation.

This work was supported in part by AFOSR (Contract No. F4920-00-C-0019), by the Alexander von Humboldt Foundation, and by the Deutsche Forschungsgemeinschaft (Schwerpunktprojekt "Group-III Nitrides"). We are grateful to J. Northrup, R. Feenstra, M. Scheffler, and O. Brandt for useful discussions and to A. da Rosa for assistance with the calculations.

[1] A. R. Smith et al., Phys. Rev. Lett. 79, 3934 (1997).

[2] J. Fritsch, O. F. Sankey, K. E. Schmidt, and J. B. Page, Phys. Rev. B 57, 15360 (1998).

[3] K. Rapcewicz, M. Buongiorno Nardelli, and J. Bernholc, Phys. Rev. B 56, R12 725 (1997).

[4] J. Elsner, M. Haugk, G. Jungnickel, and Th. Frauenheim, Solid State Commun. 106, 739 (1998).

[5] A. Munkholm et al., Phys. Rev. Lett. 83, 741 (1999).

[6] P. Hohenberg and W. Kohn, Phys. Rev. 136, B864 (1964); W. Kohn and L. J. Sham ibid. 140, A1133 (1965).

[7] M. Bockstedte, A. Kley, J. Neugebauer, and M. Scheffler, Comput. Phys. Commun. 107, 187 (1997).

[8] MOCVD growth conditions are close enough to equilibrium (as established by sufficiently high mobilities of point defects on the surface and in the bulk) for this assumption to be valid.

[9] CRC Handbook of Chemistry and Physics, edited by David R. Lide (CRC Press, Boca Raton, Florida, 1992), 73rd ed., pp. $5-18$.

[10] J.E. Northrup, J. Neugebauer, R. M. Feenstra, and A. R. Smith, Phys. Rev. B 61, 9932 (2000).

[11] M. D. Pashley, Phys. Rev. B 40, 10481 (1989).

[12] J. E. Northrup, R. Di Felice, and J. Neugebauer, Phys. Rev. B 56, R4325 (1997). 